PROCEEDINGS OF THE

AMERICAN MATHEMATICAL SOCIETY

Volume 125, Number 7, July 1997, Pages 2119-2125

S 0002-9939(97)03816-1

\title{
THE STRONG OPEN SET CONDITION IN THE RANDOM CASE
}

\author{
NORBERT PATZSCHKE
}

(Communicated by Palle E. T. Jorgensen)

\begin{abstract}
To describe some fractal properties of a self-similar set or measure, such as the Hausdorff dimension and the multifractal spectrum, it is useful that it satisfy the strong open set condition, which means there is an open set satisfying the open set condition and, additionally, a part of the self-similar set must meet the open set. It is known that in the non-random case the strong open set condition and the open set condition are equivalent. This paper treats the random case. If the open set condition is assumed, we show that there is a random open set satisfying the strong open set condition. Further, we give an application to multifractal analysis of the random self-similar fractal.
\end{abstract}

\section{Notation}

In this section we recall the definition of self-similar sets and self-similar measures, and give some properties. A more detailed introduction and the proofs of the properties may be found in $[\mathrm{PZ}]$ and in $[\mathrm{AP}]$.

Let $K \subset \mathbb{R}^{d}$ be a fixed compact set with $K=\overline{\operatorname{int} K}$. We are given a positive integer $N \geq 2$ and a probability measure $\mu$ on $\operatorname{Sim}^{N} \times[0,1]^{N}$, where $\operatorname{Sim}$ is the space of all similarities of $\mathbb{R}^{d}$ equipped with the usual topology of uniform convergence on compact sets. In this paper we assume the following.

Assumption 1. (I) $\int \sum_{i=1}^{N} p_{i} \mu\left(d\left(S_{1}, \ldots, S_{N} ; p_{1}, \ldots, p_{N}\right)\right)=1$,

(II) $S_{i}(\operatorname{int} K) \subset \operatorname{int} K$ for all $i=1, \ldots, N$ and $S_{i}(\operatorname{int} K) \cap S_{j}($ int $K)=\emptyset$ for all $i \neq j$ for $\mu-$ a. a. $\left(S_{1}, \ldots, S_{N} ; p_{1}, \ldots, p_{N}\right)$,

(III) there exist $p_{\min }>0$ and $r_{\min }>0$ such that $p_{i} \geq p_{\min }$ and Lip $S_{i} \geq r_{\min }$ for all $i=1, \ldots, N$ and $\mu-$ a. a. $\left(S_{1}, \ldots, S_{N} ; p_{1}, \ldots, p_{N}\right)$.

Condition (II) is known as the open set condition.

In the sequel we often make use of symbolic dynamics. Let $\Sigma=\{1, \ldots, N\}^{\mathbb{N}}$ be the code space over the indices $1, \ldots, N, \Sigma_{n}=\{1, \ldots, N\}^{n}$ the space of all sequences of length $n$, and $\Sigma_{*}=\bigcup_{n=0}^{\infty} \Sigma_{n}$. For $\tau \in \Sigma_{n}$ denote by $|\tau|=n$ the length of $\tau$, and by $\tau \mid k$ the truncation of $\tau$ to the first $k$ entries, $k \leq n$. For $\tau \in \Sigma_{*}$ and $\sigma \in \Sigma_{*} \cup \Sigma$ we write $\tau \prec \sigma$ if there is a $\sigma^{\prime} \in \Sigma_{*} \cup \Sigma$ with $\tau=\sigma \sigma^{\prime}$. Further, let $[\tau]=\{\sigma \in \Sigma: \tau \prec \sigma\}$ be the cylinder sets in $\Sigma, \tau \in \Sigma_{*}$.

Received by the editors January 16, 1996 and, in revised form, February 7, 1996.

1991 Mathematics Subject Classification. Primary 28A80; Secondary 60D05, 60G57.

Key words and phrases. Random fractals, (strong) open set condition, multifractals.

(C)1997 American Mathematical Society 
Define the space $\Omega=\left(\operatorname{Sim}^{N} \times[0,1]^{N}\right)^{\Sigma_{*}}$. Let $\mathfrak{F}$ be the product $\sigma$-algebra on $\Omega$. Taking $\mathbb{P}$ the product measure with $\mu$ on each component we get our primary probability space $(\Omega, \mathfrak{F}, \mathbb{P})$.

For $\omega \in \Omega$ and $\tau \in \Sigma_{*}$ write

$$
\omega(\tau)=\left(S_{\tau 1}(\omega), \ldots, S_{\tau N}(\omega) ; p_{\tau 1}(\omega), \ldots, p_{\tau N}(\omega)\right)
$$

and $S_{\emptyset}(\omega)=i d, p_{\emptyset}(\omega)=1$. By $\mathfrak{F}_{k}$ we denote the $\sigma$-algebra generated by all $S_{\tau}$ and $p_{\tau}$ with $|\tau| \leq k$. For brevity write

$$
\begin{aligned}
\bar{S}_{\tau} & =S_{\tau \mid 1} \circ \cdots S_{\tau|| \tau \mid}, \\
r_{\tau} & =\operatorname{Lip} S_{\tau} \\
\bar{r}_{\tau} & =\operatorname{Lip} \bar{S}_{\tau}=r_{\tau \mid 1} \cdots r_{\tau|| \tau \mid}, \\
\bar{p}_{\tau} & =p_{\tau \mid 1} \cdots p_{\tau|| \tau \mid}, \\
K_{\tau} & =\bar{S}_{\tau} K
\end{aligned}
$$

for $\tau \in \Sigma_{*}$.

In $[\mathrm{AP}]$ it is shown that the random variables

$$
X_{\tau}=\lim _{n \rightarrow \infty} \sum_{\eta \in \Sigma_{n}} p_{\tau(\eta \mid 1)} \cdots p_{\tau(\eta \mid n)}
$$

exist for all $\tau \in \Sigma_{*}$ and

$$
\Psi_{\omega}([\tau])=\bar{p}_{\tau}(\omega) X_{\tau}(\omega)
$$

extends to a random measure $\Psi_{\omega}$ on $\Sigma$ for $\mathbb{P}-$ a. a. $\omega \in \Omega$, with $\mathbb{E} \Psi(\Sigma)=\mathbb{E} X_{\emptyset}=1$ and $\mathbb{E} X_{\emptyset}^{2}<\infty$.

Let us define random mappings $\pi_{\omega}: \Sigma \rightarrow K$ by

$$
\pi_{\omega}(\sigma)=\lim _{n \rightarrow \infty} \bar{S}_{\sigma \mid n}(\omega)\left(x_{0}\right) .
$$

This limit exists for $\mathbb{P}$-almost all $\omega$ and does not depend on the choice of $x_{0}$.

The random measure $\Phi$ with $\Phi_{\omega}=\Psi_{\omega} \circ \pi_{\omega}^{-1}$ is called the random self-similar measure, and the random set $\Xi$ with $\Xi_{\omega}=\pi_{\omega}(\Sigma)$ the random self-similar set.

We call a random subset $\Gamma \subset \Sigma_{*}$ a Markov stopping, if

(I) for each $\sigma \in \Sigma$ and each $\omega \in \Omega$ there is a unique $\tau \in \Gamma(\omega)$ with $\tau \prec \sigma$, and

(II) $\{\omega \in \Omega: \tau \in \Gamma(\omega)\} \in \mathfrak{F}_{|\tau|}$ for all $\tau \in \Sigma_{*}$.

A simple example is the random set

$$
\Gamma_{r}=\left\{\tau \in \Sigma_{*}: \bar{r}_{\tau}<r \leq \bar{r}_{\tau|| \tau \mid-1}\right\}
$$

for $r \in(0,1)$.

If $\Gamma$ is a Markov stopping then, by the open set conditon, $\left\{\operatorname{int} K_{\tau}(\omega): \tau \in \Gamma(\omega)\right\}$ is a family of mutually disjoint sets for almost all $\omega \in \Omega$. Let $\mathfrak{F}_{\Gamma}$ be the sub- $\sigma-$ algebra of $\mathfrak{F}$ generated by $\left\{S_{\eta}, p_{\eta}\right.$ : there is a $\tau \in \Gamma$ with $\left.\eta \prec \tau\right\}$.

Further, let us introduce shift operators $\Delta_{\tau}: \Sigma_{*} \cup \Sigma \rightarrow \Sigma_{*} \cup \Sigma$ by

$$
\Delta_{\tau}(\sigma)=\tau \sigma
$$

and write $T_{\tau}(\omega)=\omega \circ \Delta_{\tau}$. Then $S_{\eta}\left(T_{\tau} \omega\right)=S_{\tau \eta}(\omega)$ and $p_{\eta}\left(T_{\tau} \omega\right)=p_{\tau \eta}(\omega)$. We will denote the objects generated by $T_{\tau}$ with a superscript $\tau$, e. g. $S_{\eta}^{\tau}(\omega)=$ $S_{\eta}\left(T_{\tau} \omega\right), \Phi_{\omega}^{\tau}=\Phi_{T_{\tau} \omega}$, and so on.

The measures $\Phi$ and $\Psi$ and the set $\Xi$ fulfill the following invariances (cf. [AP, $\mathrm{PZ}])$. 
Theorem 2. Let $\Gamma$ be a Markov stopping. Then

(i) $\Psi=\sum_{\tau \in \Gamma} \bar{p}_{\tau} \Psi^{\tau} \circ \Delta_{\tau}^{-1}$, where the $\Psi^{\tau}$ are $i . i$. d. copies of $\Psi$ and independent of $\mathfrak{F}_{\Gamma}$.

(ii) $\Phi=\sum_{\tau \in \Gamma} \bar{p}_{\tau} \Phi^{\tau} \circ \bar{S}_{\tau}^{-1}$, where the $\Phi^{\tau}$ are $i$. i. d. copies of $\Phi$ and independent of $\mathfrak{F}_{\Gamma}$.

(iii) $\Xi=\bigcup_{\tau \in \Gamma} \bar{S}_{\tau} \Xi^{\tau}$ where the $\Xi^{\tau}$ are $i$. $i$.d. copies of $\Xi$ and independent of $\mathfrak{F}_{\Gamma}$.

$\Psi, \Phi$ and $\Xi$ are characterized by the properties of the theorem above and by the requirement $\mathbb{E} \Phi\left(\mathbb{R}^{d}\right)=\mathbb{E} \Psi(\Sigma)=1$.

\section{THE MAIN THEOREM}

Now we are able to state the main theorem of this paper. The proof is analogous to one of Schief $[\mathrm{S}]$.

Theorem 3. Let the assumptions 1 be satisfied. Then there is a random open set $U$ with $U=\operatorname{int} \bar{U}$, such that the following hold.

(i) $S_{i}\left(U^{i}\right) \subset U$ for all $i=1, \ldots, N$ with probability one $\left(\right.$ where $\left.U^{i}(\omega)=U\left(T_{i} \omega\right)\right)$.

(ii) $S_{i}\left(U^{i}\right) \cap S_{j}\left(U^{j}\right)=\emptyset$ for all $i \neq j$ with probability one.

(iii) $\Xi \cap U \neq \emptyset$ with probability one.

Proof. 1. Fix $\varepsilon>0$ and let $K^{(\varepsilon)}=\bigcup_{x \in K} B(x, \varepsilon)$. For $\eta \in \Sigma_{*}$ write $G_{\eta}(\omega)=$ $\bar{S}_{\eta}(\omega)\left(K^{(\varepsilon)}\right)$ and define

$$
I(\eta)(\omega)=\left\{\tau \in \Gamma_{\bar{r}_{\eta}(\omega)}(\omega): G_{\eta}(\omega) \cap K_{\tau}(\omega) \neq \emptyset\right\} .
$$

Fix $x_{0} \in \operatorname{int} K$. Since $\operatorname{int} K$ is open and bounded, there are $0<r<R<\infty$ such that $B\left(x_{0}, r\right) \subset \operatorname{int} K \subset K \subset B\left(x_{0}, R\right)$. Let $\tau \in I(\eta)(\omega)$. By definition of $K_{\tau}$ and of $\Gamma_{\bar{r}_{\eta}(\omega)}(\omega)$,

$$
\begin{aligned}
\mathcal{L}^{\mathrm{d}}\left(K_{\tau}(\omega)\right) & \geq \bar{r}_{\tau}(\omega)^{d} \mathcal{L}^{\mathrm{d}}\left(B\left(x_{0}, r\right)\right) \\
& \geq \bar{r}_{\eta}(\omega)^{d} r_{\min }^{d} \mathcal{L}^{\mathrm{d}}\left(B\left(x_{0}, r\right)\right) .
\end{aligned}
$$

On the other hand, since $G_{\eta}(\omega) \subset B\left(\bar{S}_{\eta}(\omega) x_{0}, \bar{r}_{\eta}(\omega)(R+\varepsilon)\right)$, we have $K_{\tau}(\omega) \subset$ $B\left(\bar{S}_{\eta}(\omega) x_{0}, \bar{r}_{\eta}(\omega)(3 R+\varepsilon)\right)$. By volume estimating therefore

$$
\begin{aligned}
\# I(\eta)(\omega) \bar{r}_{\eta}(\omega)^{d} r_{\min }^{d} \mathcal{L}^{\mathrm{d}} B\left(x_{0}, r\right) & \leq \sum_{\tau \in I(\eta)(\omega)} \mathcal{L}^{\mathrm{d}}\left(K_{\tau}(\omega)\right) \\
& \leq \mathcal{L}^{\mathrm{d}}\left(B\left(\bar{S}_{\eta}(\omega) x_{0},(3 R+\varepsilon) \bar{r}_{\eta}(\omega)\right)\right) \\
& =\bar{r}_{\eta}(\omega)^{d} \mathcal{L}^{\mathrm{d}}\left(B\left(x_{0}, 3 R+\varepsilon\right)\right) .
\end{aligned}
$$

That means the cardinality of $I(\eta)(\omega)$ is bounded above, independent of $\omega \in \Omega$ and

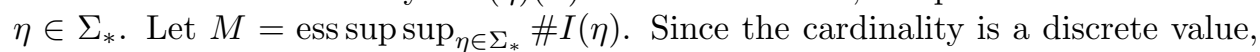
there is an $\eta_{0} \in \Sigma_{*}$ such that $\mathbb{P}\left(\# I\left(\eta_{0}\right)=M\right)>0$.

2. Take $\omega \in \Omega$ and $\eta \in \Sigma_{*}$ such that $\# I(\eta)(\omega)=M$. Let $\sigma \in \Sigma_{*}$ and $\tilde{\omega} \in$ $T_{\sigma}^{-1}\{\omega\}$. Then $\bar{r}_{\sigma \tau}(\tilde{\omega})=\bar{r}_{\sigma}(\tilde{\omega}) \bar{r}_{\tau}^{\sigma}(\tilde{\omega})=\bar{r}_{\sigma}(\tilde{\omega}) \bar{r}_{\tau}(\omega)$ and $K_{\sigma \tau}(\tilde{\omega})=\bar{S}_{\sigma}(\tilde{\omega}) K_{\tau}(\omega)$. Hence, $\sigma \tau \in I(\sigma \eta)(\tilde{\omega})$ for all $\tau \in I(\eta)(\omega)$. By maximality of $I(\eta)(\omega)$ this implies $I(\sigma \eta)(\tilde{\omega})=\sigma I(\eta)(\omega)$ and $\# I(\sigma \eta)(\tilde{\omega})=M$. 


\section{Denote}

$$
\Omega_{0}=\left\{\omega \in \Omega: \text { there is an } \eta \in \Sigma_{*} \text { with } \# I(\eta)(\omega)=M\right\} .
$$

Then $q=\mathbb{P}\left(\Omega_{0}\right)>0$. Furthermore, $T_{i}^{-1} \Omega_{0} \subset \Omega_{0}$ for all $i$ and, by definition of $\mathbb{P}$, $\left\{T_{1}^{-1} \Omega_{0}, \ldots, T_{N}^{-1} \Omega_{0}\right\}$ is an independent family. Therefore,

$$
\begin{aligned}
q & =\mathbb{P}\left(\Omega_{0}\right) \\
& \geq \mathbb{P}\left(\bigcup_{i=1}^{N} T_{i}^{-1} \Omega_{0}\right) \\
& =1-\mathbb{P}\left(\bigcap_{i=1}^{N} T_{i}^{-1} \Omega_{0}^{c}\right) \\
& =1-\mathbb{P}\left(\Omega_{0}^{c}\right)^{N} \\
& =1-(1-q)^{N}
\end{aligned}
$$

which implies (since $q>0$ ) that $q=1$. Hence, for almost all $\omega$ there is an $\eta(\omega) \in \Sigma_{*}$ such that $\# I(\eta)(\omega)=M$. The mapping $\omega \mapsto \eta(\omega)$ may be assumed to be measurable. With step 2 we get $I\left(\sigma \eta^{\sigma}(\omega)\right)(\omega)=\sigma I^{\sigma}\left(\eta^{\sigma}(\omega)\right)(\omega)$ for all $\sigma \in \Sigma_{*}$.

Denote

$$
\bar{\Omega}=\left\{\omega \in \Omega: T_{\tau} \omega \in \Omega_{0} \text { for all } \tau \in \Sigma_{*}\right\} .
$$

Since $\Sigma_{*}$ is countable and $\mathbb{P}$ a product measure we infer that $\mathbb{P}(\bar{\Omega})=1$.

4. Define

$$
U(\omega)=\bigcup_{\tau \in \Sigma_{*}} \bar{S}_{\tau}(\omega) \circ \bar{S}_{\eta^{\tau}(\omega)}^{\tau}(\omega)\left(K^{(\varepsilon / 2)}\right)
$$

for $\omega \in \bar{\Omega}$. It remains to show that the assertions hold.

(i) If $\omega \in \bar{\Omega}$ then

$$
\begin{aligned}
S_{i}(\omega)\left(U^{i}(\omega)\right) & =S_{i}(\omega)\left(\bigcup_{\tau \in \Sigma_{*}} \bar{S}_{\tau}^{i}(\omega) \circ \bar{S}_{\eta^{i \tau}(\omega)}^{i \tau}(\omega)\left(K^{(\varepsilon / 2)}\right)\right) \\
& =\bigcup_{\tau \in \Sigma_{*}} \bar{S}_{i \tau}(\omega) \circ \bar{S}_{\eta^{i \tau}(\omega)}^{i \tau}(\omega)\left(K^{(\varepsilon / 2)}\right) \\
& \subset U(\omega)
\end{aligned}
$$

for all $i=1, \ldots, N$.

(ii) Assume there are an $\omega \in \bar{\Omega}$ and a pair $i \neq j$ with $S_{i}\left(U^{i}(\omega)\right) \cap S_{j}\left(U^{j}(\omega)\right) \neq$ $\emptyset$. By definition of $U$ there are $\sigma, \tau \in \Sigma_{*}$ and $\eta=\eta^{i \sigma}(\omega)$ and $\bar{\eta}=\eta^{j \tau}(\omega)$ and $y \in \bar{S}_{i \sigma}(\omega) \circ \bar{S}_{\eta}^{i \sigma}\left(K^{(\varepsilon / 2)}\right) \cap \bar{S}_{j \tau}(\omega) \circ \bar{S}_{\bar{\eta}}^{j \tau}(\omega)\left(K^{(\varepsilon / 2)}\right)$. Without loss of generality we assume $\bar{r}_{j \tau \bar{\eta}}(\omega) \leq \bar{r}_{i \sigma \eta}(\omega)$. Choose $\tau^{\prime} \prec \tau \bar{\eta}$ such that $j \tau^{\prime} \in \Gamma_{\bar{r}_{i \sigma \eta}(\omega)}(\omega)$. By definition there are $y_{1} \in K_{i \sigma \eta}(\omega)$ with $d\left(y_{1}, y\right)<\bar{r}_{i \sigma \eta}(\omega) \cdot \varepsilon / 2$ and $y_{2} \in K_{j \tau^{\prime}}(\omega)$ with $d\left(y_{2}, y\right)<\bar{r}_{j \tau \bar{\eta}}(\omega) \cdot \varepsilon / 2<\bar{r}_{i \sigma \eta}(\omega) \cdot \varepsilon / 2$. Hence, $d\left(y_{1}, y_{2}\right)<\bar{r}_{i \sigma \eta}(\omega) \cdot \varepsilon / 2$, which implies $K_{j \tau^{\prime}}(\omega) \cap G_{i \sigma \eta}(\omega) \neq \emptyset$; hence $j \tau^{\prime} \in I\left(i \sigma \eta^{i \sigma}(\omega)\right)(\omega)$, being a contradiction to step 3.

(iii) Clearly, $K_{\eta(\omega)}(\omega) \subset U(\omega)$ for all $\omega \in \bar{\Omega}$ and, hence, $\Xi(\omega) \cap U(\omega) \neq \emptyset$.

5. Replacing $U$ by $U^{\prime}=\overline{\operatorname{int} U}$, we infer that $U^{\prime}=\overline{\operatorname{int} U^{\prime}}$. Moreover, (i), (ii), and (iii) remain valid for $U^{\prime}$, concluding the proof. 
Remark 1. In $[\mathrm{AP}]$ we said that the strong open set condition is satisfied, if the assertions of the theorem above hold with a non-random set $U$. But many proofs remain valid replacing this non-random set by a random set. There are some differences between the sets $U$ and int $K$. While $K$ is given a priori, the random set $U$ depends (in general) on the whole $\sigma$-algebra $\mathfrak{F}$. A disadvantage is also the fact that there is no constant $r_{0}$ such that for almost each $\omega$ there is a ball with radius $r_{0}$ inside $U(\omega)$.

\section{An APPLiCATion}

An application is the determination of the multifractal spectrum. Let us consider the sets

$$
\Xi_{a}=\left\{x \in \Xi: \lim _{r \downarrow 0} \frac{\log \Phi(B(x, r))}{\log r} \text { exists and equals } a\right\}
$$

of points with local dimension $a$. The multifractal spectrum is defined by the Hausdorff dimensions of these sets.

In $[\mathrm{AP}]$ we defined a function $\beta: \mathbb{R} \rightarrow \mathbb{R}$ by

$$
\mathbb{E} \sum_{i=1}^{N} p_{i}^{q} r_{i}^{\beta(q)}=1 .
$$

In $[\mathrm{F}]$ some properties of this function are listed. Let $\alpha(q)=-\beta^{\prime}(q)$. Then $\alpha$ is either constant or strictly decreasing. The multifractal spectrum is related to the Legendre transform $f=\beta^{*}$ of $\beta$ satisfying $f(\alpha(q))=q \alpha(q)+\beta(q)$. The key of the proof was the fact that $\log d(x, \partial K)$ is integrable if the strong open set condition is satisfied (cf. [AP, 2.8]). An analogue is valid in our case.

Lemma 4. Let $U$ be the random open set from Theorem 3. Then

$$
\mathbb{E} \int|\log d(x, \partial U)| \Phi(d x)<\infty
$$

Proof. By Theorem 3 there are an $\eta \in \Sigma_{*}$ and a $\delta>0$ such that $\mathbb{P}\left(d\left(K_{\eta}, \partial U\right)>\right.$ $\left.\delta / r_{\min }\right)>0$. Let $r=\operatorname{ess} \inf \bar{r}_{\eta} r_{\min }>0$ and

$$
0<z=\mathbb{E} 1\left\{d\left(K_{\eta}, \partial U\right)>r \delta / r_{\min }\right\} \Psi([\eta])<1 .
$$

Write $\Gamma(n)=\Gamma_{r^{n}}$ and

$$
G_{n}=\left\{\tau \in \Gamma(n): d\left(K_{\eta}, \partial U\right) \leq r^{n} \delta\right\} .
$$

Let $\sigma \in G_{n}$. Then $\bar{r}_{\sigma \eta} \geq r^{n+1}$. If $d\left(K_{\eta}^{\sigma}, \partial U^{\sigma}\right)>r \delta / r_{\min }$, then, since $\sigma \in \Gamma(n)$,

$$
\begin{aligned}
d\left(K_{\sigma \tau}, \partial U\right) & \geq d\left(K_{\sigma \eta}, \partial S_{\sigma} U^{\sigma}\right) \\
& =\bar{r}_{\sigma} d\left(K_{\eta}^{\sigma}, \partial U^{\sigma}\right) \\
& >r^{n+1} \delta .
\end{aligned}
$$


Hence

$$
\begin{aligned}
\mathbb{E} & {\left[\sum_{\tau \in G_{n+1}, \tau \succ \sigma} \Psi[\tau] \mid \mathfrak{F}_{|\sigma|}\right] } \\
& =\bar{p}_{\sigma} \mathbb{E}\left[\sum_{\tau \in G_{n+1}, \tau \succ \sigma} \Psi[\tau] / \bar{p}_{\sigma} \mid \mathfrak{F}_{|\sigma|}\right] \\
& \leq \bar{p}_{\sigma}\left(1-\mathbb{E}\left[\sum_{\tau \in \Gamma(n+1) \backslash G_{n+1}, \tau \succ \sigma \eta} \Psi\left(K_{\tau}\right) / \bar{p}_{\sigma} \mid \mathfrak{F}_{|\sigma|}\right]\right) \\
\leq & \bar{p}_{\sigma}\left(1-\mathbb{E}\left[1\left\{d\left(K \sigma_{\eta}, \partial U^{\sigma}\right)>r \delta / r_{\min }\right\} \Psi^{\sigma}[\eta] \mid \mathfrak{F}_{|\sigma|}\right]\right) \\
& =\bar{p}_{\sigma}(1-z) \\
& =(1-z) \mathbb{E}\left[\Psi[\sigma] \mid \mathfrak{F}_{|\sigma|}\right] .
\end{aligned}
$$

Let $\tau \in G_{n+1}$. Then there exists a unique $\sigma \in \Gamma(n)$ with $\sigma \prec \tau$. Furthermore,

$$
d\left(K_{\sigma}, \partial U\right) \leq d\left(K_{\tau}, \partial U\right) \leq r^{n+1} \delta \leq r^{n} \delta .
$$

Hence, $\sigma \in G_{n}$. This implies

$$
\mathbb{E} \sum_{\tau \in G_{n+1}} \Psi[\tau] \leq(1-z) \mathbb{E} \sum_{\sigma \in G_{n}} \Psi[\sigma]
$$

and, by induction,

$$
\mathbb{E} \sum_{\tau \in G_{n}} \Psi[\tau] \leq(1-z)^{n}
$$

for all $n$. Since $\pi^{-1}\left\{x \in \Xi: d(x, \partial U) \leq \delta r^{n}\right\} \subset \bigcup_{\tau \in G_{n}}[\tau]$,

$$
\mathbb{E} \Phi\left\{x \in \Xi: d(x, \partial U) \leq \delta r^{n}\right\} \leq \mathbb{E} \sum_{\tau \in G_{n}} \Psi[\tau] \leq(1-z)^{n},
$$

which implies the assertion.

The proof of the next theorem is, with little changes (replacing $K$ by $\bar{U}$ if necessary), the same as in [AP, 3.10]. Let $\alpha_{\min }=\inf \{\alpha: f(\alpha(q))>0\}$ and $\alpha_{\max }=\sup \{\alpha(q): f(\alpha(q))>0\}$.

Theorem 5. Let the assumptions 1 be satisfied. Then either case I or case II holds.

Case I: $\alpha_{\min }=\alpha_{\max }=D$. Then $\operatorname{dim} \Xi=\operatorname{dim} \Xi_{D}=D$ with probability one and $\Xi_{a}=\emptyset$ for all $a \neq D$ with probability one.

Case II: $\alpha_{\min }<\alpha_{\max }$. Let $a \in \mathbb{R}$, then

(i) if $a<\alpha_{\min }$ or $a>\alpha_{\max }$ then $\Xi_{a}=\emptyset$ with probability one,

(ii) if $\alpha_{\min }<a<\alpha_{\max }$ then $\operatorname{dim} \Xi_{a}=f(a)$ with probability one,

(iii) $\operatorname{dim} \Xi=f(\alpha(0))$ with probability one.

Analogous results as in [AP] hold for generalized dimensions (cf. [AP, 4.11]) and tangential distributions (cf. [AP, 5.2]). 


\section{REFERENCES}

[AP] M. Arbeiter and N. Patzschke, Random self-similar multifractals, Math. Nachr. 181 (1996), $5-42$.

[F] K. J. Falconer, The multifractal spectrum of statistically self-similar measures, J. Theoret. Probab. 7 (1994), 681-702. MR 95m:60076

[PZ] N. Patzschke and U. Zähle, Self-similar random measures IV. - The recursive construction model of Falconer, Graf, and Mauldin and Williams, Math. Nachr. 149 (1990), 285 302. MR 92j:28007

[S] A. Schief, Separation properties of self-similar sets, Proc. Amer. Math. Soc. 122 (1994), 111 - 115. MR 94k:28012

Fakultät für Mathematik und Informatik, Friedrich-Schiller-Universität Jena, D07740 Jena, Germany

E-mail address: patzschke@minet.uni-jena.de 